\title{
¿Quién elige mi dieta informativa? La datificación automatizada de personas en el ambiente digital
}

\section{Martha Vidal-Sepúlveda}

Universidad Austral de Chile. Facultad de Ingeniería. Instituto de Informática Doctorando en Comunicación, Universidad de la Frontera- Universidad Austral de Chile I martha.vidal@uach.cl / https://orcid.org/oooo-ooo2-0929-8179

\section{Cristian Olivares}

Instituto de Informática, Universidad Austral de Chile | colivares@inf.uach.cl / https://orcid.org/oooo-ooo2-4991-5784

\section{Resumen}

Desde la reflexión teórica, este trabajo analiza cómo la tecnología deviene en un dispositivo psicopolítico que configura el nuevo ambiente digital. Primero, como un espacio de flujo de información que otorga sentido a nuestra relación con el mundo y, en una segunda instancia, en la formación de nuestra identidad digital en tanto sujetos en desarrollo que se significan en la experiencia digital. En este contexto, los motores de búsqueda, como software cultural, ofrecen una dieta informativa personalizada y brindan respuestas a consultas por medio de resultados sesgados, elaborados a partir de los patrones de comportamiento de los sujetos y desmitificando, de este modo, el acceso global a la información.

\section{Who chooses my diet? The automated notification of people in the digital environment}

\begin{abstract}
From the theoretical point of view, this work analyzes how technology configures the new digital environment, as a space of information flow that gives meaning to our relationship with the world and; the development of our digital identity as subjects in development that mean in the digital experience. In this context, search engines, as cultural software, offer a personalized information diet, providing answers to queries by means of biased results, elaborated from the behavior patterns of the subjects, demystifying the global access to information.
\end{abstract}

\section{Palabras clave}

Dieta informativa Psicopolítica Alfabetización mediática Personalización y motores de búsqueda

\section{Keywords}

Information diet Digital environment Media literacy Personalization and search engines 


\section{Introducción}

En la sociedad global el avance de la tecnología informática automatiza la captura de datos sobre las personas, permitiendo la construcción de complejos bancos de datos que reconocen patrones a partir de las acciones realizadas por los sujetos biológicos en la red. Mediante algoritmos se captura, almacena y distribuye su información personal para utilizarla como insumo en el mercado de la información. La datificación algorítmica actúa con mínimas regulaciones, debido a que la opacidad de sus procedimientos dificulta su regulación y fiscalización (Palmiotto, 2021), asimismo, el poder seductor de los servicios globales sobre los sujetos biológicos les permite actuar casi sin oposición de sus usuarios. Esta opacidad de los procesos algorítmicos actúa sobre las personas y los países, afectando la democracia y libertades (Marjanovic y Cecez-Kecmanovic, 2017), en un proceso de datificación que, además, discrimina entre seres y no-seres, es decir, decide quienes son sujetos de interés en la sociedad global. Bajo este esquema existen regiones y poblaciones, dentro y fuera del primer mundo, que no son datificadas, cuyos sujetos biológicos no participan de los "beneficios" ni de las narrativas globales.

En este contexto, los motores de búsqueda emergen como un nuevo mecanismo de control y discriminación del acceso y difusión de la información. Su uso cotidiano oculta el peligro del poder hegemónico que ostentan sobre la población, especialmente la más joven. El aparente libre acceso a la información está determinado por los patrones de comportamiento que los algoritmos como dispositivo psicopolítico han elaborado a partir de nuestras acciones en la red, ofreciéndonos una dieta informativa personalizada. Por lo tanto, no existe un acceso abierto sino uno condicionado por decisiones algorítmicas, aparentemente neutrales e inocuas.

\section{La datificación de personas como insumo}

La datificación de las personas corresponde a un proceso de caracterización de los sujetos mediante procesos algorítmicos y/o estadísticos como instrumento científico de análisis para la toma de decisiones. Para Mattelart (2007: 15) el proyecto de sociedad de la información toma forma en los siglos XVII-XVIII, en los cuales la matemática representa lo racional y útil. Este proceso de datificación se iniciaría en la organización del pensamiento a partir del número en la búsqueda por objetivizar la sociedad. De acuerdo a nuestro planteamiento, la datificación, como tecnología psicopolítica, describe el comportamiento y los atributos de los individuos y los territorios, siempre que sean de utilidad para la productividad o se encuentren representados en el discurso representativo de los medios (Lodder y Loui, 2018). Debido a esto, grandes masas de población quedan marginadas o invisibilizadas del ambiente digital, bajo una lógica de inclusión/exclusión. Por consiguiente, los sujetos datificados, relevantes para los algoritmos, integran las fuerzas biológicas del ambiente digital y, por tanto, se hace necesaria su caracterización para hacer más eficiente la producción de servicios, lo que les permite controlar no solo a la población, sino también al mercado.

\section{La conformación de subjetividades en el ambiente digital}

Este nuevo ambiente digital ofrece a los individuos la posibilidad de convertirse en sujetos globales capaces de: conectarse con el mundo, obtener la información que necesitan, compartir y crear nueva información participando de las narrativas transmedia y conformando comunidades (Jenkins, Ford y Green, 2015). Si bien es 
cierto que muchas personas pueden estar de acuerdo con esta afirmación, porque en cierta medida se cumplen dichas promesas, esta promesa no es universal ni tampoco gratuita. Este nuevo ambiente es dominado por las empresas globales quienes son dueñas de la mayor parte de los servicios a los que se puede acceder en la web, pero sus transacciones son invisibles para las personas. En este ambiente se transan las acciones registradas por los sujetos biológicos como la materia prima, desde una mirada neoliberal, que es transformada y explotada y, en consecuencia, consumida. Para la conformación de los que denominaremos "sujetos datificados", la empresa global utiliza como insumos los datos de los sujetos biológicos que acceden a la red, deseosos de participar en un ambiente digital, aparentemente abierto y libre para las distintas formas de comunicación. Por su parte, los países también usan estas interacciones en internet como insumo para el control y vigilancia de sus ciudadanos, ya que la "convergencia digital de todos los datos y tecnologías también amplía al infinito las posibilidades de rastreo y colonización de las prácticas cotidianas" (Sibilia, 2005: 66). De esta forma, el ambiente digital lo domina quién disponga de la tecnología suficiente y apropiada para procesar la big data y elaborar modelos de personalización a partir de las acciones realizadas por los sujetos biológicos en sus redes.

De esta manera, los algoritmos, siguiendo un ordenamiento lógico-racional, consumen las acciones que se desprenden de las interacciones, las que se hacen cada vez más intensas por parte de los sujetos biológicos con los servicios-globales, produciendo sujetos datificados listos para ser consumidos por los algoritmos de personalización, que ordenan la vida, las reglas y la conducta de los sujetos biológicos (Just y Latzer, 2017). Podemos observar cómo servicios-globales de entretenimiento, tales como Netflix (Siles et al., 2019), domestican el comportamiento de los usuarios y los seducen a partir de estrategias de recomendación algorítmicas inferidas a partir de la datificación de las actividades cotidianas capturadas desde cualquier dispositivo. Incluso, los algoritmos y servicios globales replican y potencian los normativos de género presentes en la sociedad (Ni Loideain y Adams, 2020).

En esta inmensidad de datos, solo los algoritmos gobiernan (Sastre Domínguez y Gordo López, 2019), siendo los únicos capaces de extraer patrones y, en consecuencia, guiar el curso de las acciones cotidianas de los seres biológicos, incapaces de procesar datos masivos con diferentes estructuras y definiciones, dando paso a la gobernanza algorítmica (Pasquinelli, 2016). De esta forma, los algoritmos configuran las subjetividades digitales a partir de dichos datos, con el riesgo de amplificar los sesgos de los sujetos y de los algoritmos presentes en la web (Baeza-Yates, 2018, 2020).

En este contexto, las empresas globales seducen a las personas a interactuar con las interfaces de software, quienes están dispuestas a consumir los servicios tecnológicos de generación de información en cada una de sus actividades cotidianas, pero el acceso está condicionado por la brecha digital. Primero, por la brecha socioeconómica que limita el acceso a la conexión a internet a gran parte del territorio mundial y, en un segundo momento, por la brecha de uso, determinada por las capacidades técnicas y de comprensión de los servicios de la web (Llorca-Abad, 2012); una marginación que se acentúa al limitar su posibilidad de participación en las narrativas culturales mediáticas (García-Canclini, 2004), lo que finalmente exacerba la desigualdad social.

\section{De la subjetivación del cuerpo a las subjetividades digitales}

Tal como lo describe Cachorro "El cuerpo es un mapa, un objeto significante que adquiere sentidos y significados en sus puestas en escena" (2018:3) y, siguiendo este planteamiento, el ambiente digital es un escenario recurrente en el que participan innumerables cuerpos que comparten y nutren sus subjetividades a partir del 
contenido que producen y consumen. De esta forma, la conformación de subjetividades en el ambiente digital depende de la disposición de los sujetos biológicos a participar activamente en la red, a través de la socialización de su subjetividad contribuyendo a diluir las fronteras entre lo público y lo privado, proyectando su vida personal al ambiente digital (Sibilia, 2012). Para Han (2013: 68) "los social media y los motores de búsqueda personalizados erigen en la red un absoluto espacio cercano, en el que está eliminado el afuera". En este nuevo espacio, el sujeto biológico construye una identidad digital que se conforma a partir de sus interacciones en internet, es decir, a los datos que comparte en su red de contactos y los que su red comparte sobre él, por lo que el sujeto biológico pierde el control de su intimidad (Tello-Díaz, 2013), ya que muchas veces no tiene conciencia de los datos que ha registrado, ni tampoco tiene el dominio sobre los datos que se comparten sobre él en sus redes de contacto, puesto que se han desdibujado los límites entre lo privado y lo público, afectando a los cuerpos mediante la digitalización de la vida (Sibilia, 2005). Así, a medida que consumimos los servicios globales, vamos dando forma a nuestra huella digital, la que permanece en la red sin un control sobre quién o quiénes pueden ver nuestros datos ni tampoco los posibles usos de nuestra información (Bazarova y Choi, 2014). Sin embargo, a nuestra identidad biológica le corresponden distintas identidades digitales, construidas a partir de los datos que ha capturado cada uno de los servicios globales que consumimos en la red, complejizando la relación indisociable entre nuestro cuerpo y nuestra subjetividad (Cachorro, 2008). De esta manera, a partir de un mismo sujeto biológico es posible construir varias versiones datificadas, según el algoritmo y/o el servicio global al que se somete. Por consiguiente, este ambiente digital se constituye de millones de individuos digitales aislados pero conectados. Este homo digitalis descrito por Han (2014a) es un ser expuesto que solicita atención, pero con una identidad disuelta en la masa, incapaz de congregarse ni oponerse al poder, sino tan solo en movimientos fugaces e inestables. Según su propuesta, no existiría una clase dominante que explote a este sujeto digital, sino que es el mismo quien se explota y se expone.

Siguiendo la tesis de autoexplotación de Han, la participación activa del ser biológico en la red supone un consentimiento, según el cual el ser biológico vende su individualidad como materia prima a los intereses del mercado de los grandes datos, a cambio de un servicio. Sin embargo, en esta operación de consumo de la oferta global en el ambiente digital no existe una simetría de poder entre los sujetos biológicos y las empresas globales. Es más, planteamos que se accede de manera coercitiva a la construcción de un sujeto datificado por cada servicio global que se consume, ya que muchos de los servicios-globales se ofertan en modalidad gratuita o bajo demanda, relevando como atributos de su negocio la flexibilidad y adaptación a las necesidades e intereses de sus clientes. Además, negarse a la datificación implica la pérdida de servicios globales o bien, la reducción de su calidad y, en consecuencia, ser marginado de las narrativas globales.

Asimismo, a pesar de este supuesto consentimiento, el sujeto biológico desconoce los alcances y las múltiples reconstrucciones a las que es sometido cada vez que realiza acciones en la web y, a su vez, le es imposible caracterizar sus múltiples posibilidades de existencia datificada, desconociendo los trayectos de sus fragmentadas subjetividades (Cachorro, 2008) las que son consumidas por la ciencia de datos para ofertar servicios a la sociedad digitalizada (Igual y Seguí, 2017).

En consecuencia, los algoritmos operan como dispositivos psicopolíticos digitales (Han, 2014b; Vásquez, 2016) que gobiernan por medio de la lectura de patrones utilizados para elaborar predicciones de la conducta de los sujetos biológicos, a partir de sus preferencias en la red. En tiempos de pandemia, el miedo e incertidumbre han facilitado que se incrementen los mecanismos de vigilancia con la complacencia 
de los sujetos biológicos, a través de dispositivos tecnológicos que capturan datos asociados a la trazabilidad de los enfermos de COVID-19 (Chen et al., 2021; Ho et al., 2021). De este modo, la psicopolítica opera en la web como "técnica de dominación que estabiliza y reproduce el sistema dominante por medio de una programación y control psicológico" (Han 2014b: 117). Esta sofisticada técnica de poder ya no necesita ejercer la fuerza sobre los cuerpos, sino que el control lo alcanza explotando las emociones humanas a través de la seducción, el análisis y la vigilancia transparente (Han, 2013) de los sujetos datificados.

\section{Los motores de búsqueda de información como herramienta para la creación de subjetividades}

Actualmente, el acceso a la información se encuentra mediado por una tecnología dominada por las empresas globales y algunos estados hegemónicos - tales como Rusia y China, que cuentan con sus propios motores de búsqueda - colonizando los mercados y la vida cotidiana de todos los sujetos conectados a la red (Li y Dong, 2020). El motor de búsqueda es un software cultural que permite acceder a información indexada en la web y, como tal, corresponde al soporte de acciones culturales, es decir, el motor de búsqueda permite, entre otras acciones:

crear, intercambiar y acceder a artefactos culturales que contienen representaciones e ideas, creencias y valores estéticos (...); crear y compartir información y conocimiento en línea (...); participar en la ecología de información en línea mediante preferencias y metadatos (...). (Manovich, 2013: 21).

Específicamente, los motores de búsqueda surgen como herramientas creadas para el intercambio de información y conocimiento. Este software cultural ha alcanzado una alta penetración en ámbitos empresariales, educativos y personales y es capaz de ofrecer respuestas a innumerables tipos de consultas, por lo que es una de las aplicaciones de mayor éxito en la red. Estos motores (Google, Bing, Ask, Aol, DuckDuckGo, etc.) pertenecen a empresas globales que poseen índices a la mayor cantidad de información que ha sido posible reunir en la historia de la humanidad (Rasmussen, 2003) y, que los sujetos biológicos disponemos gratuitamente para su distribución a partir de los patrones de selección y exploración de documentos en la web, en una visión utópica del acceso a la información global.

Se suele pensar que al emitir una consulta en un motor de búsqueda es posible acceder a toda la información disponible en la red, sin embargo, consultar a un motor de búsqueda implica la selección de una porción de información de acuerdo a los criterios de la búsqueda, el comportamiento histórico y los mecanismos de indexación (Steiner et al., 2020). Además, no es posible hablar de una red única y abierta, sino que de acuerdo a su acceso, se la ha clasificado en: internet global, la cual es accesible mediante buscadores y protocolos de uso; internet invisible o profunda, cuya información no tiene indexación automática y es necesario conocer la dirección URL (Fornas, 2003); y la internet oscura, compuesta por redes restringidas, servidores y redes privadas accesibles mediante autorización o softwares específicos (Gehl, 2014). De acuerdo a esto, el acceso a la información en línea no es global, sino que depende de variables de acceso tecnológico y humano.

Sin embargo, los motores de búsqueda permiten recuperar información disponible en la denominada internet global a través de la vinculación entre los documentos o recursos electrónicos indexados y las consultas emitidas por los sujetos biológicos, según su relevancia (Page et al., 1999), pero la cercanía de los resultados de búsqueda con la consulta emitida dependen de variables tecnológicas y humanas, 
las que al combinarse complejizan el proceso de búsqueda de información. Se debe tener en cuenta que en el proceso de búsqueda, en la interacción entre la tecnología y el ser biológico intervienen las variables cognitivas, físicas y afectivas de estos últimos (Kuhlthau, 1991), así como a las capacidades de la propia tecnología para responder a las necesidades de búsqueda (Marchionini, 2006).

Dichos algoritmos surgen como una alternativa evolucionista y determinista tecnológica que busca incrementar la eficiencia de las búsquedas frente a la creciente cantidad de información de la nube, disminuyendo el agobio de cientos de resultados con información diversa. La complejidad técnica de los servicios de búsqueda se reduce por medio de algoritmos que investigan los patrones históricos y geolocalizan a los sujetos datificados entregando recomendaciones de consultas (Duarte Torres, et al., 2012), detectando el propósito del usuario (Sadikov et al., 2010; Santos, et al., 2003) o elaborando un ranking que ordena los resultados de la búsqueda considerando criterios tales como la relevancia, la diversidad y la popularidad y el comportamiento histórico del usuario (Balabantaray, 2017; Xing y Ghorbani, 2004), restringiendo y ordenando los resultados de búsqueda a partir de esos filtros (Yoganarasimhan, 2020). Es así, como se ha determinado que los algoritmos seleccionan las respuestas que recibimos de acuerdo a nuestros clics, nuestro idioma y la popularidad del sitio (Jiang, 2014a). En esta ecuación, también suma nuestra ubicación geográfica, denominada por Jiang como "búsqueda provincialista" (Jiang 2014a; 2014b; Cano-Orón, 2019). Asimismo, también está condicionada por la agenda mediática de cada país (Cano- Orón, 2019), la que en países como Chile, es concentrada y controlada por dos grupos económicos. En definitiva, la eficiencia del motor de búsqueda se alcanza mediante la prohibición de acceso a grandes porciones de la web a través de operaciones heurísticas que descartan caminos de exploración y, con ello, introducen sesgos (Baeza-Yates, 2020) en los resultados.

Para la elaboración de estos modelos, las empresas globales utilizan los patrones de comportamiento de los sujetos datificados con el propósito de dirigir su comportamiento durante la búsqueda y alcanzar mayores niveles de precisión. Los algoritmos hurgan en el banco de datos de sujetos datificados, impactando en las decisiones, en este caso, informativas de los sujetos biológicos. Pero la opacidad de las decisiones algorítmicas (Pasquale, 2015), impiden conocer con exactitud las decisiones del modelo inferido desde el algoritmo, por lo tanto, se ignoran las reglas algorítmicas aplicadas sobre los datos y, en particular, las que seleccionan nuestros resultados en el motor de búsqueda.

Si bien, la empresa global opera en el mundo de los datos a través de algoritmos, los modelos computacionales inferidos desde datos masivos tienen efecto en el mundo biológico de los sujetos a partir de la interpretación subjetiva de los patrones observados, los que modifican su comportamiento (Chander, 2016; Boyd, Levy y Marwick, 2014) y, por tanto, su cultura (Siles et al., 2019), conformando lo que proponemos denominar dieta informativa personalizada. Pero, una dieta informativa dirigida por nuestros patrones de búsqueda no solo restringe el acceso a la información, sino que modela nuestra construcción de subjetividades. Así, la psicopolítica actúa por medio del tráfico de información saturada y filtrada que recibimos diariamente, siendo capaz de generar imaginarios culturales basados en la emoción que reproduce el sistema dominante (Han, 2014b).

En consecuencia, el sesgo de los motores de búsqueda, a través de su ranking de resultados, establece las fronteras de la información, y determina quién tiene o no acceso a cierta información, según esto, estar conectado a internet no es suficiente para asegurar un acceso global a la información. Por lo tanto, la personalización de 
los servicios-globales de búsqueda no solo reduce el espacio de búsqueda con filtros que determinan los vínculos que, supuestamente, se acercan a nuestros intereses o áreas de estudio, sino que también nos alejan de la diversidad, dejando fuera toda la información antagónica o contradictoria al patrón del sujeto datificado, temporal y geográficamente.

En este espacio virtual, opera también el poder de la empresa global, determinando la información a la que los seres biológicos tienen o no acceso; una lógica de poder seductora que se muestra amigable y accesible, ya que los seres biológicos parecen no sospechar de la información recibida y mantienen una creencia de que, a partir de la búsqueda, se pueden alcanzar todos los resultados posibles o, supuestamente, los más relevantes. Sin embargo, se ha establecido que los algoritmos de filtro crean una burbuja alrededor de los seres biológicos que limita la oportunidad de acceso a información diversa, su acceso se limita a los sitios que consulta frecuentemente $u$ otros similares (Parisier, 2017). Esto es potenciado por los sesgos cognitivos propios de los sujetos al momento de interactuar con los motores de búsqueda (Baeza-Yates, 2018). Por lo tanto, si el ser datificado nunca o casi nunca explora fuentes de calidad, se reduce su posibilidad de acceder a ellas cuando lo requiera, exacerbando la subjetividad construida a partir de algoritmos y decisiones sesgadas.

Finalmente, la búsqueda de información en la web es una habilidad fundamental en la alfabetización mediática y el activismo de datos que lucha por reducir el control de los algoritmos (Sastre Domínguez y Gordo López, 2019), sin embargo, la mayoría de los seres biológicos no cuenta con las habilidades necesarias para acceder eficientemente a la información (Druin et al., 2009; Qureshi et al., 2015; Şendurur y Yildirim, 2015). Incluso se ha observado una baja tendencia a la utilización de funciones avanzadas en los motores de búsqueda (Yamamoto et al., 2018). De esta forma, el escaso desempeño en esta competencia reduce la eficiencia y efectividad del proceso de búsqueda e impacta en el estado de ánimo de los usuarios (Foo et al., 2014; Rosman, Mayer y Krampen, 2015), dejando muchas decisiones cotidianas a cargo de los algoritmos.

Por lo tanto, la información en la nube no es accesible para toda la población, para su acceso no solo se necesita estar conectado, sino que se requieren habilidades críticas que permitan a los seres biológicos interactuar con la información disponible y, a la vez, habilidades técnicas para estresar al algoritmo y alcanzar hallazgos insospechados.

\section{Conclusión}

La sociedad global ha modificado las relaciones de poder entre los países y los sujetos, lo que se evidencia en acciones colectivas que han sido capaces de intervenir en la agenda, pero también ha encumbrado a la empresa-global a un nuevo estatus de poder, basado en su capacidad de concentrar y distribuir la información global. Bajo este marco, es urgente considerar los riesgos éticos y legales a los que se ven expuestos los sujetos y los países.

Es necesario reflexionar y tomar acciones frente a la amenaza a la democracia y la reducción de libertades de las personas. El avance de la datificación de personas por medio del uso de servicios-globales, ha permitido concentrar datos personales de los sujetos, en sí mismos sin un valor aparente, pero que son el principio para elaborar sofisticadas bases de datos, a partir la fusión de distintas variables que les permiten obtener información nueva y valiosa para controlar distintos mercados y/o ofertar nuevos productos; es decir, nuestra individualidad, basada en nuestros datos, preferencias, rutinas y deseos, ya no es personal ni de nuestra propiedad. Es más, toda nuestra 
información disponible en la red es posible de ser: copiada y distribuida por terceros, analizada por algoritmos vigilantes y accesible mediante los motores de búsqueda.

Hoy está en riesgo la libertad y autonomía de las personas para tomar decisiones, desde la selección de un documento en un motor de búsqueda, la compra de bienes y servicios, hasta su influencia en procesos de elecciones. Las personas no tenemos la certeza si nuestra selección está basada en nuestra propia decisión, o si ésta ha sido condicionada por la dieta informativa personalizada elaborada por un algoritmo.

Es por ello que para conquistar la libertad y proteger la democracia en el mundo global, las personas requieren de competencias críticas que les permitan reflexionar sobre sus acciones en el mundo virtual, entendiendo este acceso global como una posibilidad cierta de obtener un mayor acceso a información y de participar democráticamente en las narrativas globales. Una actitud crítica que le permita comprender que el acceso no es global, sino que la globalidad está subordinada a la acción algorítmica que registra, almacena y analiza nuestra información a partir de nuestras acciones en internet.

Por consiguiente, la alfabetización digital es una necesidad y no puede remitirse a la educación formal sino que debe ser abierta y extenderse a lo largo de la vida. Las personas deben disponer de competencias críticas y técnicas para proteger su individualidad, cuidando su información personal y colectiva pero, asimismo, apropiarse de los espacios virtuales para elaborar mensajes propios que representen los saberes de las diferentes culturas, actuando en resistencia al poder ejercido por las empresas de las comunicaciones (Castells, 2009). De esta forma, la alfabetización mediática no puede ser reducida al consumo crítico de medios, ya que una participación plena en la cultura digital implica la producción y participación activa de sus miembros. Sin embargo, en nuestra realidad nacional y en el mundo en general, existen amplios territorios desconectados por su pobreza o aislamiento, cuyos sujetos biológicos no participan de las narrativas digitales. Además, entre los grupos conectados existen deficiencias en las competencias técnicas y de lectura de medios, ambas condiciones necesarias para una participación activa en la cultura de la convergencia mediática.

En consecuencia, la configuración del ambiente digital es desigual y parcial, por lo que la información que puede ser recuperada mediante los motores de búsqueda representa el conocimiento acumulado y la cultura de una pequeña parte de la población mundial. Sumado a esto, para mejorar nuestra experiencia de búsqueda, los modelos algorítmicos seleccionan la porción de datos que se adapta a nuestro perfil, ofreciéndonos una dieta informativa acrítica, eficiente y lista para nuestro consumo. 


\section{Q Referencias bibliográficas}

"Baeza-Yates, Ricardo. 2018. Bias on the web. En Communications of the ACM. Vol. 61, no. 6, 54- 61. <https://doi.org/10.1145/3209581>

»Baeza-Yates, Ricardo. 2020, Bias in search and recommender systems. En Fourteenth ACM Conference on Recommender Systems (RecSys '20). p. 2. <https: // doi.org/https://doi.org/10.1145/3383313.3418435>

» Balabantaray, Rakesh. 2017. Evaluation of web search engine based on ranking of results and its features. En International Journal of Information and Communication Technology. Vol.10, no. 4, 392- 405. <https://doi.org/10.1504/ IJICT.2017.084337>

»Bazarova, Natalya y Yoon Hyung Choi. 2014. Self-disclosure in social media: Extending the functional approach to disclosure motivations and characteristics on social network sites. En Journal of Communication. Vol. 64, no.4, 635-657. <https://doi.org/10.1111/jcom.12106>

"Boyd, Dana, Karen Levy y Alice Marwick. 2014. The networked nature of algorithmic discrimination. En Data and Discrimination: Collected Essays. Open Technology Institute. <http://www.danah.org/papers/2014/DataDiscrimination.pdf> [Consulta: 10 agosto 2019].

»Cachorro, Gabriel. 2008. Cuerpo y subjetividad: Rasgos, configuraciones y proyecciones. En Jornadas de Cuerpo y Cultura de la UNLP. Universidad Nacional de La Plata. Facultad de Humanidades y Ciencias de la Educación. Departamento de Educación Física. <http://www.memoria.fahce.unlp.edu.ar/trab_eventos/ ev.697/ev.697.pdf> [Consulta: 10 agosto 2019].

"Cano-Orón, Lorena. 2019. Dr. Google, what can you tell me about homeopathy? Comparative study of the top1o websites in the United States, United Kingdom, France, Mexico and Spain. En El profesional de la información. Vol. 28, no. 2. $<$ https://doi.org/10.3145/epi.2019.mar.13>

»Castells, Manuel. 2009. Comunicación y Poder. Madrid: Alianza.

"Chander, Anupam. 2016. The racist algorithm. En Michigan Law Review. Vol. 115, no. 6. <http://repository.law.umich.edu/mlr/vol115/iss6/13> [Consulta: 8 agosto 2019].

» Chen, Hung-Jui, Lin, Hung-Jung, Wu, Mei-Chen, Tang, Hung-Jen, Su, Bo-An, y Lai, Chin-Cheng. 2021. The implementation of an active surveillance integrating information technology and drive-through coronavirus testing station for suspected COVID-19 cases. En Journal of Infection. Vol. 82, no. 2, 282-327.

"Druin, Allison, Elizabeth Foss, Leshell Hatley, Evan Golub, Mona Leigh Guha, Jerry Fails y Hilary Hutchinson. 2009. How children search the internet with keyword interfaces. En IDC'09: Proceedings of the 8th International Conference on Interaction Design and Children. p. 89-96. <https://doi.org/10.1145/1551788.1551804>

"Duarte Torres, Sergio, Djoerd Hiemstra, Ingmar Weber y Pavel Serdyukov. 2012. Query recommendation for children. En CIKM '12: Proceedings of the 21st ACM international conference on Information and knowledge management. p. 20102014. ACM. <https://doi.org/10.1145/2396761.2398562>

" Foo, Schubert, Shaheen Majid, Intan Azura Mokhtar, Xue Zhang, Yung-Ke Chang, Brendan Luyt y Yin-Leng Theng. 2014. Information literacy skills of se- 
condary school students in Singapore. En Aslib Journal of Information Management. Vol. 66, no.1, 54-76. <https://doi.org/10.1108/AJIM-08-2012-0066>

"Fornas Carrasco, Ricardo. 2003. La cara oculta de Internet (en línea). En Hipertext.net. No. 1. <http://goo.gl/G6DMkh> [Consulta: 8 agosto 2019].

»García-Canclini, Néstor. 2004. Diferentes, desiguales y desconectados: mapas de la interculturalidad. Barcelona: Gedisa.

"Gehl, Robert. 2014. Power/freedom on the dark web: A digital ethnography of the Dark Web Social Network. En New Media \& Society. Vol.18, no. 7, 1219-1235. < https://doi.org/10.1177/1461444814554900>

»Han, Byung-Chul. 2013. La Sociedad de la Transparencia. Barcelona: Herder.

»Han, Byung-Chul. 2014a. En el Enjambre. Barcelona: Herder.

»Han, Byung-Chul. 2014b. Psicopolítica. Barcelona: Herder.

» Ho, H. J., Wei Yang Lim, Brenda Ang y Amanda Chow. 2021. Use of surveillance technology to enhance exposure management for healthcare workers during the COVID-19 pandemic. En The Journal of Hospital Infection. Vol. 107, 101-02. <https://doi.org/10.1016/j.jhin.2020.09.024 0195-6701>

» Igual, Laura y Santi Seguí. 2017. Introduction to Data Science. En Introduction to Data Science: A Python Approach to Concepts, Techniques and Applications. Chan: Springer. p. 1-4.

» Jenkins, Henry, Sam Ford y Joshua Green. 2015. Cultura transmedia: la creación de contenido y valor en una cultura en red. Barcelona: GEDISA.

" Jiang, Min. 2014a. Search concentration, bias, and parochialism: A comparative study of Google, Baidu, and Jike's search results from China. En Journal of communication. Vol. 64, no. 6, 1088-1110. <https://doi.org/10.1111/jcom.12126>

" Jiang, Min. 2014b. The business and politics of search engines: A comparative study of Baidu and Google's search results of Internet events in China. En New media \& society. Vol.16, no. 2, 212-233. <DOI: 10.1177/1461444813481196>

" Just, Natascha y Michael Latzer. 2017. Governance by algorithms: reality construction by algorithmic selection on the Internet. En Media, Culture \& Society. Vol.39, no. 2, 238- 258. <https://doi.org/10.1177/0163443716643157>

"Kuhlthau, Carol. 1991. Inside the search process: Information seeking from the user's perspective. En Journal of the American Society for Information. Vol.42, no. 5, 361- 371. <https://doi.org/10.1002/(SICl)1097-4571(199106)42:5<361::AIDASI6>3.0.CO;2-\%23>

" Li, Xiaohui y Hongbin Dong. 2020. An Oligopoly Two-Stage-Game Model for Investigating the Search Engine Market. En International Journal of Performability Engineering. Vol. 16, no. 1, 1781-1792. <https://doi.org/ doi: 10.23940/ijpe.20.11. p10.17811792>

» Llorca-Abad, Germán. 2012. Exclusión digital y límites de la comunicación mediada (en línea). En Trípodos. Vol.31, 111-123. <http://goo.gl/aCLasz > [Consulta: 12 agosto 2019]

» Lodder, Arno, y Ronald Loui. 2018. Data algorithms and privacy in surveillance: On stages, numbers and the human factor. En Barfield W. y Pagallo U, ed. Research handbook on the law of artificial intelligence. Edward Elgar Publishing. p. 375-384. 
» Manovich, Lev. 2013 [2012]. El Software toma el mando. Traducción de Software Takes Command (versión del 30 de septiembre de 2012, publicada bajo licencia Creative Commons en manovich.net) por Everardo Reyes-García.

" Marchionini, Gary. 2006. Exploratory search: from finding to understanding. En Communications of the ACM. Vol. 49, no. 4, 41- 46. < https://www.inf.unibz. it/ ricci/lSR/papers/p41-marchionini.pdf> [Consulta: 27 junio 2020].

" Marjanovic, Olivera y Dubravka Cecez-Kecmanovic. 2017. Exploring the tension between transparency and datification effects of open government IS through the lens of Complex Adaptive Systems. En The Journal of Strategic Information Systems. Vol. 26, no. 3, 210-232.

" Mattelart, Armand. 2007. Historia de la sociedad de la información. Barcelona: Paidós.

»Ni Loideain, Nóra y Rachel Adams. 2020. From Alexa to Siri and the GDPR: the gendering of virtual personal assistants and the role of data protection impact assessments. En Computer Law \& Security Review. Vol. 36, 105366. <https://doi. org/10.1016/j.clsr.2019.105366>

"Page, Lawrence y Sergey Brin, Rajeev Motwani y Terry Winograd. 1999. The PageRank Citation Ranking: Bringing Order to the Web. Technical Report. Stanford InfoLab. <http://ilpubs.stanford.edu:809o/422/> [Consulta: 27 junio 2020].

»Palmiotto, Francesca. 2021. The Black Box on Trial: The Impact of Algorithmic Opacity on Fair Trial Rights in Criminal Proceedings. En Ebers M., Cantero Gamito M., eds. Algorithmic Governance and Governance of Algorithms. Cham: Springer. p. 49-70. < https://doi.org/10.1007/978-3-030-50559-2_3>

"Pariser, Eli. 2017. El filtro burbuja: Cómo la web decide lo que leemos y lo que pensamos. Barcelona: Taurus.

»Pasquale, Frank. 2015. The black box society. Londres: Harvard University Press.

»Pasquinelli, Matteo. 2016. The spike: On the growth and form of pattern police. En Stephanie Hankey, Marek Tuszynski and Anselm Franke, eds. Nervous Systems. Berlin: HKW/ Spector books. p. 276-291.

"Qureshi, Ambrina, Syed Bokhari, Madiha Pirvani y Narendar Dawani. 2015. Understanding and practice of evidence based search strategy among postgraduate dental students: a preliminary study. En The Journal of Evidence-Based Dental Practice. Vol. 15, no. 2, 44-49. <https://doi.org/10.1016/j.jebdp.2014.08.002 >

"Rasmussen, Edie. 2003. Indexing and retrieval for the Web. En Annual Review of Information Science and Technology (ARIST). Vol. 37, 91-124.

» Rosman, Tom, Anne-Kathrin Mayer y Günter Krampen. 2015. Combining selfassessments and achievement tests in information literacy assessment: empirical results and recommendations for practice. En Assessment $\&$ Evaluation in Higher Education. Vol. 40, no. 5, 740-754.

»Sadikov, Eldar, Jayant Madhavan, Lu Wang y Alon Halevy. 2010. Clustering query refinements by user intent. En WWW '10: Proceedings of the 19th international conference on World wide web. p. 841-850. <https://doi. org/10.1145/1772690.1772776>

»Santos Jr, Eugene, Hien Nguyen, Qunhua Zhao y Hua Wang. 2003. User modelling for intent prediction in information analysis. En Proceedings of the Human Factors and Ergonomics Society Annual Meeting. Vol. 47, no. 8, 1034-1038. Sage CA: Los Angeles, <https://doi.org/10.1177/154193120304700818> 
»Sastre Domínguez, Paz y Ángel Juan Gordo López. 2019. Data activism versus algorithmic control. New governance models, old asymmetries. En Revista Científica de Información y Comunicación. No. 16, 157-182.

"Şendurur, Emine y Zahide Yildirim. 2015. Students' Web Search Strategies With Different Task Types: An Eye-Tracking Study. International Journal of Human-Computer Interaction. Vol. 31, no. 2, 101-111. <https://doi.or g/10.1080/10447318.2014.959105>

"Sibilia, Paula. 2005. El hombre postorgánico: cuerpo, subjetividad y tecnologías digitales. Argentina: FCE.

»Sibilia, Paula. 2012. La intimidad como espectáculo. Argentina: FCE.

» Siles, Ignacio, Johan Espinoza-Rojas, Adrián Naranjo y María Fernanda Tristán. 2019. The mutual domestication of users and algorithmic recommendations on Netflix. En Communication, Culture \& Critique. Vol. 12, no. 4, 499-518.

»Steiner, Miriam, Melanie Magin, Birgit Stark y Stefan Geiß. 2020. Seek and you shall find? A content analysis on the diversity of five search engines' results on political queries. En Information, Communication \& Society. 1-25. <https://doi.or g/10.1080/1369118X.2020.1776367>

» Tello-Díaz, Lucía. 2013. Intimidad y "extimidad» en las redes sociales. Las demarcaciones éticas de Facebook. En Comunicar. Vol. 21, no. 41, 205-213. <http:// dx.doi.org/10.3916/C41-2013-20>

»Vásquez Rocca, Adolfo. 2016. Byung-Chul Han: la sociedad de la transparencia, psicopolítica y autoexplotación neoliberal: de lo viral-inmunológico a lo neuronal-estresante. En Reflexiones Marginales. No. 31. < https://reflexionesmarginales.com.mx/blog/2016/01/29/byung-chul-han-2/>

»Xing, Wenpu y Ali Ghorbani. 2004. Weighted pagerank algorithm. En Proceedings. Second Annual Conference on Communication Networks and Services Research, IEEE. p. 305-314.. <DOI: 10.1109/DNSR.2004.1344743>

» Yoganarasimhan, H. 2020. Search personalization using machine learning. En Management Science. Vol. 66, no. 3, 1045-1070.< https://doi.org/10.1287/ mnsc.2018.3255>

"Yamamoto, Yusuke, Takehiro Yamamoto, Hiroaki Ohshima y Hiroshi Kawakami. 2018. Web access literacy scale to evaluate how critically users can browse and search for web information. En WebSci '18: Proceedings of the 1oth ACM Conference on Web Science p. 97-106. <https://doi.org/10.1145/3201064.3201072> 